\title{
IgM Monoclonal Gammopathy
}

National Cancer Institute

\section{Source}

National Cancer Institute. IgM Monoclonal Gammopathy. NCI Thesaurus. Code C128812.

An abnormal laboratory test result indicating the presence of Ig M monoclonal immunoglobulin in the blood or urine. 